\title{
Relaciones públicas y comunicación para un entorno de crisis. El caso de las universidades andaluzas
}

\author{
Antonio MARÍN RuIZ \\ Universidad de Granada \\ amarin@ugr.es \\ Ángeles DURÁN MAÑES \\ Centro de Educación Superior Alberta Giménez \\ Francisco Fernández Beltrán \\ Universitat Jaume I
}

\begin{abstract}
Resumen:
Los gabinetes de comunicación universitarios nacieron en España a partir de los pasados años 80, sin haber pasado por la experiencia previa como servicios de relaciones públicas de otros países occidentales. El artículo aborda el crecimiento de funciones de los actuales servicios de comunicación, particularmente en las universidades andaluzas; así como la irrupción de las TIC como elementos que plantean la necesidad de volver sobre el concepto de servicios de relaciones públicas al servicio de instituciones en crisis.
\end{abstract}

Palabras clave: Relaciones públicas; servicio de comunicación; Internet; redes sociales; universidades andaluzas.

Public relations and communication for an environmental crisis: the case of Andalusian universities

\begin{abstract}
:
The university press offices arose in Spain in the 80s. However, those Spanish offices did not experience the evolution from public relations services into university press offices as in other Western countries. In this article, the growth of functions of existing communication services are discussed, particularly the case of Andalusian universities, as well as the emergence of ICT as elements that suggest the need for the redefinition of the concept of public relations services of institutions in crisis.
\end{abstract}

Key Words: Public relations; communication service; Internet; social networks; Andalusian universities.

\section{Referencia normalizada:}

Marín Ruiz, A. Durán Mañes, A. y Fernández Beltrán, F. (2014): Relaciones públicas y comunicación para un entorno de crisis. El caso de las universidades andaluzas. Historia y Comunicación Social. Vol. 19. Núm. Especial Marzo. Págs. 139-151.

Sumario: 1. Introducción. 2. Relaciones públicas y comunicación. 3. Nuevos servicios de comunicación, el caso español. 4. TIC y servicios de comunicación universitarios en España. 5. Los servicios de comunicación de las universidades andaluzas. 6. Conclusiones. 


\section{Introducción}

Los gabinetes de prensa o servicios de comunicación se aparecieron hace relativamente poco tiempo, no más allá de la década de los 80 del siglo pasado. La literatura científica sobre el tema apunta que se crearon con anterioridad en los países occidentales, especialmente a lo largo del fuerte crecimiento económico de los 50, bajo la denominación de oficina de relaciones públicas.

Una aparición tan tardía en España está motivada en razones económicas, en un entorno productivo y comercial muy débil; así como en el hecho de que no eran necesarios servicios que mediaran entre las instituciones y los medios de comunicación cuando aquéllas eran las únicas fuentes de información impuestas por el régimen político.

Por otra parte, el caso de las universidades cuenta con particularidades especificas: la falta de competencia entre ellas, en de un contexto de crecimiento general acelerado. De hecho, los "gabinetes de prensa" universitarios no aparecen en hasta mediados los 80 , siendo en los 90 cuando se produce su generalización, vinculada a la necesidad de atender las relaciones universidad/medios informativos. Este artículo aborda el caso de los servicios de comunicación de las universidades andaluzas específicamente, y su evolución en un período marcado por algunos factores comunes al resto del Estado:

- La creación de nuevas universidades a partir de los antiguos colegios universitarios.

- El incremento de funciones de las que hubieron de hacerse cargo los servicios de comunicación, producto de la creciente complejidad universitaria.

- Un primer impacto de las tecnologías de la información y la comunicación, particularmente del ordenador personal y del fax.

- Un segundo impacto de las TIC, ligado a la irrupción de Internet; en especial las salas de prensa digitales, el correo electrónico y las redes sociales y sus usos para la comunicación interna y externa.

En el artículo, finalmente, hacemos propuesta para un cambio necesario en la forma de abordar las funciones de los servicios de comunicación, ligada a la descripción de las que ya se desarrollan o están llamados a desempeñar estos servicios; y ello en el marco de la tradición de las relaciones públicas del ámbito anglosajón.

\section{Relaciones públicas y comunicación}

Los servicios de comunicación aparecieron tarde en nuestro país y su desarrollo, salvo en contadas excepciones, no alcanzó a los mundos de la empresa y las instituciones hasta la década de los 80 del siglo pasado. No fue así en el mundo occidental 
(particularmente en EEUU), en el que el origen de los servicios de comunicación estuvo ligado a la fundación de las relaciones públicas como disciplina regulada en el primer tercio del siglo XX. Castillo (2004: 44) señala que el norteamericano Ivy Lee fundó la profesión del "relaciones públicas" al servicio de la defensa de los intereses de la familia Rockefeller ${ }^{1}$, aunque fuera más tarde, también en Estados Unidos, Edward L. Bernays fue fundador de las RRPP como profesión ${ }^{2}$.

Las relaciones públicas "surgen cuando una determinada sociedad adquiere plena conciencia de que existe una necesidad de intercomunicación social y, al mismo tiempo, de que el modelo presuntamente idóneo para satisfacerla exige unos conocimientos o aptitudes y un esfuerzo susceptibles de constituir una profesión autónoma", Solano (1999: 98). Sería ésta la sociedad en la que Bernays desarrolla su labor como asesor y como docente, en un contexto en el que la sociedad de EEUU demanda comunicadores capaces de atender las relaciones con el conjunto de la sociedad y, particularmente, de los medios.

Castillo (2004: 45-56) dibuja una secuencia temporal en la que la figura del comunicador relaciones públicas se desarrolla en un proceso ligado a acontecimientos históricos de especial relevancia. Son cuatro los períodos a través de los cuales evolucionará esta nueva figura profesional:

- Desde finales del XIX hasta la I Guerra Mundial aparece la figura de un responsable de hacer un seguimiento de la imagen de personas y empresas en la prensa; así como, en el caso de las instituciones específicamente, en el análisis de la opinión pública ante los esfuerzos bélicos y la creación de campañas de propaganda en favor de los mismos.

- Tras la Gran Guerra, hasta la Gran Depresión, frente a los excesos de la etapa anterior, se produce una reacción contraria a la fuerza mostrada por la propaganda, al tiempo que las relaciones públicas aparecen como disciplina universitaria. Es el período en el que la figura de Edward L. Bernays define teóricamente las relaciones públicas, que van más allá de la anterior concepción de la comunicación, para trabajar con criterios científico-académicos.

- Desde la Crisis del 29 hasta el final de la II Guerra Mundial, las relaciones públicas se extienden como esfuerzo comunicativo orientado tanto a movilizar a la población en favor de los esfuerzos para la paz, en el llamado New Deal en EEUU, como a apoyar los sacrificios necesarios para ganar la II Guerra Mundial.

- Desde el final de la Guerra hasta finales del siglo XX: todos los ámbitos de la comunicación se universalizan en el mundo occidental, en formas que nos resultan ya muy familiares: servicios de comunicación/prensa, información, corporate, relaciones públicas, publicidad, marketing, fundraising, protocolo, patrocinio, mecenazgo, etc.

Se trata, ésta última, de una etapa muy larga, tanto por el período de tiempo que abarca Castillo en su descripción, como porque no alcanza a describir lo sucedido 
tras la irrupción de internet. Apuntamos, pues, una quinta etapa: 1) en ella aparece toda una gama de funciones comunicativas que corresponden a especialidades que representan, por sí mismas, tantas profesiones como las que acaban de señalarse; y 2) hablar de medios implica ya hacer referencia a un mundo que se ha hecho extremadamente complejo (prensa escrita, radio, TV e Internet -1.0, 2.0 y 3.0-, la irrupción de las redes sociales -RRSS-) y 3) las profesiones y los profesionales se multiplican, permaneciendo ya sólo en el mundo entorno de habla inglesa o cultura comunicadora anglosajona el concepto "relaciones públicas" para referirse a todos los ámbitos de la comunicación, como sucede en el caso de las universidades ${ }^{3}$.

\section{Nuevos servicios de comunicación, el caso español}

Como se señaló en la Introducción, las razones de una aparición tardía de las relaciones públicas y de la comunicación en España habría que buscarlas tanto en motivaciones económicas, derivadas de una escasa necesidad durante el Franquismo de una comunicación corporativa empresarial en un entorno productivo y comercial muy débil; y de la realidad sociopolítica de un régimen que entendía la comunicación como propaganda, haciendo de las fuentes oficiales las únicas a las que podían dirigirse los medios. Siendo por ello poco atractivo, o innecesario, contar con servicios que hemos descrito en el apartado anterior. No hubo espacio empresarial o institucional para la aparición de la figura de relaciones públicas o comunicadores en ninguno de los ámbitos antes descritos, salvo en el de la propaganda. El atraso y el aislamiento de España afectan a todos los ámbitos de la vida nacional, en un país asolado por la crisis del 98, la existencia de mercados muy reducidos que dan lugar a un pobre tejido industrial tras la pérdida del imperio colonial (mercados apenas animados por la neutralidad en la I Gran Guerra), la dictadura de Primo, el corto intermedio de la II República y la España de Franco ${ }^{4}$.

Pero la necesidad de otra forma de comunicar y de relacionarse existe y es conocida. Así, Ortega ${ }^{5}$ (1930) resume en pocas palabras a qué nos referimos en alusión a los medios y a la Universidad, cuando apunta qué habría de hacerse desde ella para contribuir a vivificar la sociedad: "La Universidad tiene que estar también abierta a la plena actualidad; más aún: tiene que estar en medio de ella, sumergida en ella".

Tímidamente a final de los años 50 empieza a poder hablarse de relaciones públicas o de comunicación en España. Barquero (1999) y Almansa $(2003,2004)$ ofrecen una visión muy amplia y básicamente coincidente sobre la literatura científica referida a este tema. Almansa realiza detallados estudios sobre la historia de los servicios de comunicación en España (2003, 8-14) y Andalucía (2004, 102-108). Para ambos, antes del nacimiento de los servicios de prensa ha de hacerse referencia a la profesión de relaciones públicas, que aparece en España en los años 50 y 60, abriendo camino a la primera expansión de los 70 . La Transición romperá el monopolio informativo que hasta entonces se había ejercido por el Estado, los medios de comunicación 
pueden acceder a cualquier fuente de noticias. Ante medios de comunicación que podían recurrir a múltiples fuentes informativas institucionales, empresariales o a otras organizaciones sociales se produjo la reacción de empresas, ayuntamientos, administraciones, sindicatos, partidos, universidades, etc. que crearon servicios dirigidos particularmente a atender las relaciones como los medios informativos desde los incipientes "gabinetes de prensa".

En nuestro país, pues, se llega tarde a la fase descrita en el apartado "d", pero lo hace como un torrente del que guardamos ya memoria los autores de este trabajo, como personas que empezaban a prestar servicios en los primeros gabinetes de prensa. Así llamados inicialmente porque se orientaban sólo a atender las peticiones de información de los medios y porque las técnicas que se empleaban eran las propias de la prensa escrita. En todos los casos, el punto de inflexión en las relaciones informativas del día a día con los medios y su transformación vino marcado por la aparición y la popularización de los fax. El hecho de que en un par de horas pudieran remitirse las notas de prensa desde los gabinetes a la redacción de decenas de medios informativos condicionó las relaciones, en tanto que el tempo cambió y las relaciones interpersonales habituales tendieron a retroceder. Lo que sucedió a continuación fue simplemente que el tiempo se pudo aprovechar mejor en los gabinetes de prensa, el número de noticias creció y la necesidad o la posibilidad de asumir nuevas funciones. Del lado de los medios se produjo una invasión de noticias y de llamadas relacionadas con ellas, se redujo el número de páginas, pero se amplió el número de noticias, creció el tamaño de la letra, se incrementó el tamaño de las imágenes. Internet nos encontró preparados parcialmente. La web 1.0, el correo electrónico, el blog, las redes sociales, los dispositivos móviles, el crecimiento exponencial de medios o la tendencia a la universalización del acceso a Internet terminaron de romper el mundo de lo que un día se pudo llamar "gabinete de prensa" y había tenido su origen en los servicios de relaciones públicas.

\section{TIC y servicios de comunicación universitarios en españa}

La última década ha visto la aparición de un amplio número de trabajos centrados en las funciones de los servicios de comunicación universitarios; con una creciente atención al impacto de tecnologías de la información y la comunicación (TIC). Así, Aguilera et al. (2010), Cuena (2005), Durán Mañes (2005), Fernández Beltrán (2007 y 2011), García Orta (2012), Losada Díaz (2002), Marín Ruiz (2012), Paniagua (2013), Pedreño (2010), Regalado (2011), Rodríguez Ruibal y Santamaría (2011), Sánchez Valle (2005) y un largo etcétera de universitarios han analizado ya parte de una experiencia de la comunicación universitaria, en la que el entorno digital representa ya un hecho; que corresponde a dos momentos radicalmente diferentes: el previo y el posterior a la aparición de las redes sociales (RRSS). Pasemos a analizar esta afirmación y qué implicaciones tiene. 
Durán Mañes (2005) y Fernández Beltrán (2007) publicaron sendas tesis doctorales en la Universitat Jaume I de Castellón centradas en el estudio y el desarrollo del corporate universitario.

Durán realizó un estudio sistemático de las universidades españolas en relación con las funciones vinculadas a la comunicación corporativa. A partir de él, se pudo afirmar que la Universidad española, en general, desarrollaba ampliamente el programa de relaciones informativas en el momento de estudio (2003-2005): mantenían un estrecho contacto con los medios de comunicación. Por otra parte, los servicios de de comunicación solían gestionar la comunicación interna sin una planificación enmarcada dentro de planes específicos, en tanto que la comunicación corporativa era un elemento inexistente. En lo referido a la comunicación de crisis y la comunicación del rector, el estudio detectó que eran actividades que se integraban, más en la teoría que en la práctica, en los servicios de comunicación universitarios, también sin una planificación previa real y explicitada. El protocolo, como elemento indispensable en actos oficiales, se desarrollaba, pero en departamentos diferentes al de comunicación. Además, el estudio detalló la existencia de medios de comunicación propios en las universidades, generalmente publicaciones impresas; radios universitarias en una veintena de universidades y una presencia muy escasa de televisiones universitarias.

El análisis de funciones de los servicios de comunicaciones de las universidades españolas culminó con el estudio de dos cuestiones clave generalmente no resueltas en nuestros días: a) La gestión de publicidad en medios externos y en medios propios, algo que se hacía desde los servicios estudiados en todos los casos, aunque sin ninguna idea de generación de ingresos a partir de los medios propios, ni ninguna otra idea de explotación del patrocinio estratégico u otros métodos de captación de recursos. b) La gestión de la identidad visual corporativa de la propia institución, el diseño y la edición de publicaciones menores (carteles, folletos, etc.) y la coordinación de actos promocionales no figuraban, mayoritariamente, entre las funciones de los servicios de comunicación.

Fernández Beltrán (2007 y 2011) ha realizado diversos estudios referidos a la función de los portales corporativos en las universidades y a la influencia de la web 2.0 en la gestión de la comunicación interna. Cabe destacar, quizá, su planteamiento sobre la cuestión de la Internet 2.0 desde la perspectiva de la comunicación corporativa, concebida como elemento estratégico que atraviesa transversalmente la vida universitaria. En este sentido, plantea la necesidad de un urgente cambio de cultura digital en la comunicación universitaria, que se fundamentaría en el reconocimiento de las herramientas 2.0 como instrumentos para abrirse al entorno y ofrecer información de interés social, así como de conocer mejor los públicos internos y externos para conseguir la máxima eficacia institucional. Las propuestas de Fernández Beltrán, pasado más de un lustro desde el estudio de Durán, muestran que las carencias por ella detectadas encuentran parte de sus soluciones en una integración de las novedades TIC. 
Es ésta una cuestión de particular relevancia para el objeto de este trabajo, ya que la situación actual, al inicio del curso 2013-2014, difiere radicalmente de la descrita en la "Introducción", cuando se hizo referencia a que los gabinetes de prensa empezaron a aparecer y se desarrollaron en un momento en que había poca o ninguna competencia entre las universidades, en un contexto de crecimiento constante de alumnado y fuentes de financiación. Inicialmente, la aparición de Internet a mediados de los 90 apenas modificó la forma de trabajar de los que aún podían ser denominados gabinetes de prensa, propia de las redacciones de los medios. Pero la rápida evolución socioeconómica, los cambios a que se ve abocado el mundo universitario y el desarrollo de las TIC obligan a abordar cambios en una línea que nos devuelve a lo "viejo", en cierta medida al origen de las relaciones públicas o a la asunción de la interpretación inglesa de esta expresión ${ }^{6}$.

En efecto, las funciones de las que hubieron de hacerse cargo los gabinetes de prensa los transformaron, de hecho, en servicios de comunicación obligados a abordar la gestión de nuevos medios susceptibles de uso interno y externo (web 2.0, redes sociales, blog, TV online para usos informativos y formativos), la importancia creciente de la divulgación especializada, cuyo origen está en instancias universitarias muy alejadas de la dirección, la organización de eventos, la necesidad de colaborar en el diseño y la difusión de campañas de captación de recursos, la asesoría especializada al rector y a otros miembros del consejo de dirección, la preocupación por la imagen de marca, etc.

Cabe señalar, finalmente, que los cambios que precisan abordar los servicios de comunicación vienen condicionados por un competitivo contexto universitario nacional e internacional, los compromisos de España con el Espacio Europeo de Educación Superior (EEES), el panorama nacional de crisis moral y financiera o la creciente demanda social a la Universidad pública de que se implique más con la marcha general del país y rinda cuentas de su quehacer.

\section{Los servicios de comunicación de las universidades andaluzas}

Almansa Martínez (2003) y García Orta (2012) han realizado importantes aportaciones al estudio de los servicios de comunicación de las universidades andaluzas. Éstas difieren poco de lo señalado más arriba en los estudios de Fernández Beltrán o Durán, así como las de autores como de Aguilera $^{7}$ (2010). Almansa, como hiciera Durán, describe en detalle los servicios de comunicación tal como funcionaron desde su origen hasta el momento de la extensión de las TIC, en unos entornos universitarios descritos por Orta en lo referido a la comunicación orientada a públicos internos, en línea muy similar a la de Fernández Beltrán.

A partir del marco descrito por estos estudios, el análisis de la situación actual de los servicios de comunicación de las universidades andaluzas muestra que se ha producido un avance considerable en cuando a asunción de funciones de comunica- 
ción interna y externa, en la línea del concepto de relaciones públicas apuntado más arriba.

Yendo a los datos concretos, cabe señala que Andalucía cuenta en la actualidad con nueve universidades públicas: Almería, Cádiz, Córdoba, Granada, Huelva, Jaén, Málaga, Pablo de Olavide y Sevilla. Cada una de ellas cuenta con servicios de comunicación $^{8}$ con denominaciones y adscripciones diversas que, resumidamente, responden a los nombres de "Gabinete de Comunicación" o "Dirección de Comunicación", si bien en algunos se añaden los términos "y relaciones sociales" (Almería) "y Marketing" (Cádiz), o han integrado a otro servicio, como ocurre en Granada con el "Gabinete de Documentación, Edición e Información" o en Málaga con el Servicio de Comunicación e Información”.

Las funciones de los servicios están recogidas en un solo caso, el de Almería y de manera muy genérica, en los Estatutos de la Universidad, siendo lo común que los planes estratégicos de las universidades establezcan objetivos estratégicos en materia de comunicación universitaria. Objetivos que posteriormente dieron lugar a cartas de servicios integradas en los planes de calidad de las universidades.

Las funciones detalladas en las cartas de servicios no difieren, inicialmente, de lo citado por Durán Mañes, a las que se añaden algunas propias del devenir de la extensión de las TIC. Así, se citan actividades como: relación con los medios a través de la remisión de texto e imagen (en los nueve casos), gestión de la publicidad institucional (en ocho casos), elaboración del dossier de prensa (en los nueve casos), elaboración de una revista digital (en ocho casos, entendiendo por tal la sala de prensa del servicio), administración de redes sociales (8 casos), etc. Pero las diferencias llegan cuando se citan funciones esenciales en la comunicación institucional (interna y externa), como administración de las lista de distribución (correo institucional, sólo en tres casos), archivo fotográfico (dos casos), elaboración de guía de expertos (dos casos), administración de la web institucional (tres casos), protocolo (un caso), colaboración en la gestión de la mejora de la calidad de los servicios de la universidad (dos casos) o gestión de la imagen corporativa (tres casos).

La realización de estas funciones corresponde, ahora sí, a un personal que se cita sin las prevenciones que hubo a mediados de la pasada década, como síntoma de la nueva transparencia en la gestión de personal en las universidades. Así, dependiendo de las funciones atribuidas a cada servicio de comunicación, el tamaño de la universidad en cuestión o el uso o no de personal de apoyo en prácticas, el número oscila entre en 2 en el caso de Huelva ${ }^{9}$ y 19 personas en el caso de la Universidad de Málaga $^{10}$.

Una diferencia radical entre 2005 y la actualidad se encuentra en la dirección de los servicios de comunicación, si entonces ésta recaía en todos los casos en personal de administración y servicios, ahora lo hace en profesorado en seis de los casos. La interpretación de este hecho no es simple y se antoja que puede haber ventajas evidentes de este hecho, por la pertenencia del profesorado al equipo de gobierno del rector, frente al único inconveniente evidente, que radicaría en que los servi- 
cios pudieran verse alterados en su continuidad para el caso de que no hubiese una adecuada delegación de funciones en el PAS que, en todo caso, heredará la gestión del personal interino que, en este caso, es el profesorado.

\section{Conclusiones}

Los servicios de comunicación universitarios, desde su origen hasta nuestros días, han evolucionado acumulando funciones al servicio de sus instituciones. La situación actual de crisis es comparable a otros momentos descritos a lo largo del artículo, en los que la reacción ante las dificultades fue siempre confiar en profesionales de la comunicación (public relations) la gestión de información y de la imagen de la institución.

Los servicios de comunicación de las universidades andaluzas han evolucionado de manera similar a los del resto de España en cuanto a su modelo de organización y su profesionalización, a falta de estudios actuales sobre nuevas funciones para el conjunto del Estado, la tendencia en las universidades andaluzas conduce a una cierta homogeneización a partir de la publicación de sus cartas de servicios.

Conocida la historia y mirando al entorno de crisis universitaria actual en el que competencia, restricciones e imposibilidad de atender presentes y nuevas necesidades son ya hechos ¿en qué dirección habrían de evolucionar los servicios de comunicación universitarios?

La literatura científica citada en el artículo apunta a estudios sobre el uso de las redes sociales, el impacto de las TIC, la imagen de marca, el diálogo con los públicos internos, la extensión de lo audiovisual, etc. Podrían citarse decenas de estudios más, la mayoría de ellos abordan aspectos parciales de un problema que, a nuestro juicio, se resume en la siguiente síntesis:

- El concepto "public relations" ha de integrarse en la cultura organizacional, como elemento vertebrador de las actividades distribuidas entre servicios de información y comunicación; al modo altamente profesionalizado de las universidades anglosajonas, que integran todos los flujos de información operativa interna y externa.

- Los servicios de Comunicación están llamados a asumir formalmente nuevas funciones que, en algunos casos, ya vienen realizando en forma de perfiles en las redes sociales, seguimiento y evaluación de los flujos de información interna y externa, conversión de la información en comunicación (diálogo con los públicos internos y externos), transparencia informativa, etc.

- El punto de partida ha de ser que al servicio de comunicación se le asigne la responsabilidad sobre la comunicación interna como "(1) herramienta de dirección y administración (permitiendo el intercambio de información opera- 
tiva); (2) vehículo de la cultura corporativa (facilitando la distribución de la filosofía de la organización); e (3) instrumento de comunicación externa (ayudando a proyectar hacia el interior y hacia el exterior una misma imagen de la entidad)." 11

- Los servicios de comunicación han de participar en las políticas de captación de recursos externos, no limitándose a la gestión de las noticias sobre esta temática, sino implicándose en la gestación de los planes de comunicación, en su elaboración y despliegue.

- Las potencialidades de las TIC no han sido aún suficientemente reconocidas en las universidades. Corresponde a los servicios de comunicación, al menos en parte, demostrar la rentabilidad de inversiones mínimas en productos audiovisuales susceptibles de ser utilizados tanto en la docencia como en la divulgación y en la captación de alumnado, en el aula presencial o en las redes sociales, en las plataformas virtuales propias o en repositorios externos.

- En relación con el punto anterior, el uso de las RRSS es ya esencial en la comunicación corporativa universitaria ${ }^{12}$, si bien está llamado a crecer en un proceso de fragmentación de públicos objetivos a los que dirigir información cada vez más ajustada a sus intereses.

¿Cómo hacer esto posible en el conjunto de instituciones instaladas cómodamente en la web $1.0^{13}$ ? En un entorno de crisis, es casi obligado que servicios como el de Comunicación estén llamados a asumir parcelas más amplias de la gestión de la imagen y de la política de relaciones públicas de las universidades. Es éste el camino que habrán de seguir los rectorados, siguiendo una lógica que antes llevó de hablar de «gabinetes de prensa»a «servicios de comunicación», cuando se entendió que ésta era un factor estratégico; en el momento actual se ha de plantear, por coherencia y eficacia en la gestión, la necesidad estratégica de contar con servicios que integren el amplio abanico de competencias citado.

\section{Bibliografía}

\subsection{Libros}

ALMANSA MARTÍNEZ, Ana (2003) Teoría, estructura y funcionamiento de los gabinetes de comunicación. El caso andaluz. Tesis doctoral Universidad de Málaga, 2003.

ÁlVAREZ, T. y CABALLERO, M. (1997): Vendedores de imagen. Los retos de los nuevos gabinetes de comunicación, Paidós, Barcelona.

BARQUERO CABRERO, J.D. (coord.) (1999): Manual de Relaciones Públicas empresariales e institucionales. Gestión 2000, Barcelona. 
LOSADA DÍAZ, José C. (2002). Prensa e imagen corporativa en la Universidad: los públicos internos, Universidad Católica San Antonio de Murcia, Murcia. p. 148.

7.2 Capítulos o artículos en libros o revistas en papel

CUENA BOTRÁN, Pilar (2005). La dirección de comunicación en el ámbito universitario. En Marín Ruiz, A. et al. (coods.), Mass Media y Universidad. Granada.

FERNÁNDEZ BELTRÁN, Francisco (2011). Comunicación interna 2.0. La gestión de portales corporativos y redes sociales. Ed. Ciencias Sociales. Madrid.

7.3 Artículos en publicaciones web

AGUILERA MOYANO, Miguel (2010) La comunicación universitaria. En: Revista Icono 14. Año 8, Vol. 2. Madrid: pp. 90-124. http://dialnet.unirioja.es/descarga/ articulo/3301663.pdf [Consultado el 15 de diciembre de 2011]

ALMANSA MARTÍNEZ, Ana (2004) Historia de los gabinetes de comunicación en España. Revista Historia y Comunicación Social. Madrid 2004, 9, 5-21. http://revistas.ucm.es/index.php/HICS/article/view/HICS0404110005A/19269 [Consultado el 12 de septiembre de 2013]

BRITO, Julio G. et al. (2012). El uso de redes sociales por parte de las universidades a nivel institucional. Un estudio comparativo. RED Revista de Educación a Distancia, núm. 32. Murcia. http://www.um.es/ead/red/32/laaser_et_al.pdf

CASTILLO ESPARCIA, Antonio (2004) "Investigación sobre la evolución histórica de las relaciones públicas", Revista Historia y Comunicación Social. Madrid. http://revistas.ucm.es/index.php/HICS/article/view/HICS0404110043A/19277 [Consultado el 12 de septiembre de 2013]

DURÁN MAÑES, Ángeles (2005). Nuevas tendencias en la comunicación corporativa. Aplicación a un modelo de corporate universitario, tesis doctoral, Universitat Jaume I, Castellón, 2005. http://repositori.uji.es/xmlui/handle/10803/10472?locale-attribute $=$ en [Consultado el 20 de agosto 2013]

GARCÍA ORTA, Juan (2012). Comunicación interna y Universidad: una aproximación teórica. Trabajo de Fin de Master presentado para optar al Titulo de Master Universitario en Comunicación Institucional y Política. Universidad de Sevilla. http://fama2.us.es/fco/tmaster/tmaster34.pdf [Consultado el 13 de abril de 2013]

MARIN RUIZ, Antonio (2012). Retos de los servicios de comunicación ante la Universidad 2.0. En: adComunica. Revista Científica de Estrategias, Tendencias e Innovación en Comunicación, $\mathrm{n}^{\circ} 4$. Castellón: Asociación para el Desarrollo de la Comunicación adComunica, Castellón. DOI: http://dx.doi.org/10.6035/21740992.2012.3.X [Consultado el 20 de agosto de 2013]

ORTEGA Y GASSET, José (1930). La misión de la Universidad, edición electrónica a cargo de Raúl J. A. Palma, Buenos Aires, accesible desde http://www.esi2. us.es/ fabio/mision.pdf. [Consultado el 25 de septiembre de 2013]

PANIAGUA ROJANO, Francisco J. et al. (2013). La incorporación de los departamentos de comunicación de las universidades españolas al entorno digital. Un análisis cuantitativo. Estudios sobre el Mensaje Periodístico. Madrid. http:// 
revistas.ucm.es/index.php/ESMP/article/view/40948 [Consultado el 20 de agosto 2013]

PEDRENTO MUÑOZ, Andrés (2010): ¿Qué puede ser la Universidad 2.0?: visión y estrategias de actuación. http://utopias-realidades.blogspot.com.es/2009/08/ universidad-20.html [Consultado el 15 de diciembre de 2011]

REGALADO, Octavio. ¿Cómo deberían usar las Universidades las Redes Sociales? Por|Publicado: NOVIEMBRE 20, 2011.http://www.dosensocial.com/2011/11/20/ como-deberian-usar-las-universidades-las-redes-sociales/ [Consultado el 20 de agosto 2013]

RODRÍGUEZ RUIBAL, Antonio, y SANTAMARÍA CRISTINO, Patricia (2012). Análisis del uso de las redes sociales en internet: Facebook y Twitter en las Universidades españolas. Icono14 10 (2), 228-246, doi: http://10.7195/ri14.v10i2.198. [Consultado el 8 de diciembre de 2012]

\section{Notas}

1 En 1914, John Rockefeller Jr. contrató a Lee con el encargo de contrarrestar la hostilidad de los medios causada por la represión a los huelguistas en una de la empresas de la familia, que produjo la muerte de 28 trabajadores.

2 Autor en 1923 del primer libro sobre relaciones públicas, bajo el título "Cristalizando a la opinión pública".

3 Tal es el caso, por ejemplo, de la Asociación de Servicios de Comunicación y Relaciones Públicas de Universidades Europeas (EUPRIO en sus siglas en inglés).

4 Particularmente interesante resulta la lectura de las descripciones que sobre la economía de la época referida hacen, región a región y sector económico a sector económico, García de Cortazar y González Vesta, en su "Breve historia de España” (Alianza Ed. 1994, pp. 528-603).

5 La lectura del capítulo final de su "La misión de la universidad" resulta tan llamativa como el resto de la obra, tanto por su eterna actualidad como por cómo describe el reto, también de presente, de que los universitarios participen de la vida pública a través de los medios de comunicación.

6 Algo que se evidencia en la definición de EUPRIO, Association of European Public Relations and Informatiton Officers (euprio.eu), en la que se insertan los servicios de comunicación de las universidades españolas integrados en la Asociación Universitaria de Gabinetes de Comunicación (AUGAC, www.augac.es/).

7 Miguel de Aguilera supo integrar en la Universidad de Málaga todas las áreas que afectan a la comunicación interna y externa.

8 Universidad de Almería http://cms.ual.es/UAL/universidad/organosgobierno/gabcomunicacion. Universidad de Cádiz http://www.uca.es/gabcomunicacion. Universidad de Córdoba http://www.uco.es/servicios/comunicacion. Universidad de Granada http://www. ugr.es/pages/servicios/gc. Universidad de Huelva http://www.uhu.es/canaluhu. Universidad de Jaén http://www10.ujaen.es/conocenos/organos-gobierno/vicplan/comunicacion/ gcomunicacion. Universidad de Málga http://www.uma.es/servicio-comunicacion. Univer- 
sidad Pablo Olavide http://www.upo.es/comunicacion/utc. Universidad de Sevilla http:// www.comunicacion.us.es

9 Con sólo funciones de relación con los medios de comunicación y protocolo.

10 Número que se explica sobradamente porque en el Secretariado de Comunicación se integran gabinete de prensa, diseño gráfico, medios audiovisuales, servicios de información al público y hasta centralitas telefónicas de los campus.

11Fernández Beltrán, Francisco (2011). Comunicación interna 2.0. La gestión de portales corporativos y redes sociales. Madrid: Ed. Ciencias Sociales, p.204.

12 Proliferan todo tipo de "ranking" en el mundo universitario, entre ellos sobre el de uso de las RRSS. Los estudios de Regalado (2011), Brito (2012) o Rodríguez Ruibal y Santamaría (2012), por ejemplo, se centran en aspectos cuantitativos y cualitativos. Son los primeros los que llaman más la atención, en tanto que ofrecen estadísticas en forma de clasificaciones fáciles de consultar; sin embargo, el meollo de la cuestión es el cualitativo: qué se está haciendo y cómo se habrían de utilizar las redes sociales en las universidades, caracterizadas por una enorme variedad de públicos internos y externos a los que se puede llegar vía RRSS, pero sólo a partir de esfuerzos que van mucho más allá de lo que las estadísticas apenas llegan a dejar ver.

13 Crucial para el conjunto del mundo universitario la ya citada aportación del Prof. Pedreño (exrector de la Universidad de Alicante y exdirector de Universia). A este mismo punto se ha referido Marín Ruiz (2012). 